\title{
Effects of Intersection Lighting Design on Nighttime Visual Performance of Drivers
}

Rajaram Bhagavathula

Virginia Tech Transportation Institute

3500 Transportation Research Plaza, Blacksburg, VA 24061

Phone: (540) 231-5209; Fax: (540) 231-1555; Email: Rbhagavathula@vtti.vt.edu

Ronald B. Gibbons

Virginia Tech Transportation Institute

3500 Transportation Research Plaza, Blacksburg, VA 24061

Phone: (540) 231-1581; Fax: (540) 231-1555; Email: Rgibbons@vtti.vt.edu

Maury A. Nussbaum

Grado Department of Industrial \& Systems Engineering,

521 Whittemore Hall (MC 0118), 1185 Perry Street, Blacksburg, VA 24061

Phone: (540) 231-6053; Fax: (540) 231-3322; Email: nussbaum@vt.edu

Keywords: Intersection Lighting Design, Visual Performance, Night Driving, Safety, Intersection Lighting Design Standards, Rural Intersections, Isolated Intersections. 


\begin{abstract}
Nighttime crashes at intersections present a major traffic safety issue in the United States. Existing approach to intersection lighting design does not account for a driver's visual performance or the potential interactive effects of vehicle headlamps and roadway lighting. For effective design lighting at intersection, empirical research is required to evaluate the effects of lighting configuration (part of the intersection illuminated) and lighting levels on nighttime driver visual performance. The current study had two goals. First, to quantify visual performance in three lighting configurations (illuminating the intersection box, approach, or both). Second, to determine what lighting levels within each lighting configuration support the best visual performance. The study involved a target detection task, completed at night on a realistic roadway intersection. Illuminating the intersection box led to superior visual performance, as indicated by longer target detection distances, fewer missed targets, and more targets identified within a safe stopping distance. For this lighting configuration, visual performance plateaued between 7 and 10 lux of mean intersection illuminance. These results have important implications for the design of intersection lighting at isolated/rural intersections, specifically that illuminating the intersection box is an effective strategy to increase nighttime visual performance for a wider range of driver ages and could also be an energy efficient solution.
\end{abstract}

\title{
Introduction
}

Crashes at intersections constitute a disproportionate share of the total number of roadway crashes making them a major safety issue for drivers and vulnerable road users like pedestrian, bicyclists etc. For example in the United States, in 2013, intersection crashes constituted over $45 \%$ of number of crashes and $25 \%$ of the number of fatalities in the United States (NHTSA 2014). Furthermore, night crashes and fatalities account for approximately 40\% of the total crashes and fatalities at intersections (NHTSA 2014). To safely navigate an intersection, drivers should take into consideration a number of factors such as the presence of others vehicles, pedestrians, bicyclists, signal phases or presence of stop signs, etc. Indeed, intersections are one of the most complex roadway types that drivers encounter. For example, an intersection of two streets with two-way traffic on each has a total of 16 vehicle-to-vehicle 
conflict points and 16 pedestrian-to-vehicle conflict points (Turner et al. 2006). Therefore, hazard detection (pedestrian, cyclist, fixed object, another vehicle etc.) by driver is of paramount importance at intersections especially at night.

Lighting of intersections has received attention as a potential method for reducing the number of night crashes and related fatalities because it increases the amount of visual information available to the driver approaching an intersection and assists in hazard detection. Simply having lighting at intersections does appear to reduce the number of night crashes, by 13 to $45 \%$ (Bullough et al. 2013, Donnell et al. 2010, Isebrands et al. 2006, Smadi et al. 2011, Wortman and Lipinski 1974), and an increase in illuminance by 1 lux lowers night-to-day crash ratios and rates by roughly 7\% (Bhagavathula et al. 2015) and 9\% (Edwards 2015), respectively. Furthermore, Oya et al. (2002) reported that a mean roadway illuminance of $20 \mathrm{~lx}$ or more is an effective countermeasure against crashes and mean road-surface illuminance of $30 \mathrm{~lx}$ results in a statistically significant reduction of night crashes. Minoshima et al. (2006) used subjective ratings of intersection visibility to assess three intersection lighting designs (approach, corner (or box) and both approach and corner) at three illuminances (5, 10 and 15 lux). The authors concluded that at illuminances greater than 10 lux mean ratings of visibility were high in all the three lighting designs. While both Oya, Ando and Kanoshima (2002) and Minoshima, Oka, Ikehara and Inukai (2006) explored two different aspects of driver behavior, both studies indicate that greater than 10 lux of mean roadway illuminance is required to achieve enhanced perceptions of visibility and reduction in crashes. A reduction in crashes required higher illuminance as crashes often have multiple causal factors beyond just visibility.

Intersection lighting has also been given a special consideration by both the Illumination 
Engineering Society of North America (IESNA) and the Commission Internationale de l'Éclairage (CIE). These organizations have recommended minimum lighting levels for intersections, with specific levels depending on a number of factors such as roadway classification (only IESNA), speed, traffic volume, and traffic composition (only CIE). Recommended light levels for intersections, though, differ substantially from those recommended for lighting of roadways. IESNA's RP-8 recommends that the lighting level at intersections should be equal to the sum of the lighting levels of each road at the intersection (IESNA 2005). The recommended average horizontal illuminance by RP-8 ranges from 8 - 34 lux based on the pedestrian classification and the functional classification of the roads forming the intersection. CIE's 115:2010 recommends that lighting level of the intersection should always be higher than the highest lighting level of the roads that form the intersection (CIE 2010). The recommended average horizontal illuminance by CIE 115: 2010 ranges from 7.5 - 50 lux depending on the lighting classification of the road: C0 (highest light level)-C5 (lowest light level). The CIE's lighting classification of the road depends on a number of factors such as speed, traffic volume, and traffic composition. Of note, these standards provide only recommended luminance and illuminance (light incident on the roadway) levels (CIE 2010, IESNA 2005) and do not specify which parts of the intersection should be illuminated. Further, these recommended levels are a result of consensus between researchers and practitioners in the field of roadway illumination who studied the effects of roadway lighting on night crashes. Both, the process of selecting the part of the intersection that is to be illuminated and the required level of illumination for an intersection are not backed by empirical research.

Furthermore, crash data, such as the number of crashes or night-to-day crash ratios, have typically been used to assess the effectiveness of intersection lighting designs, specifically the, 
part of the intersection that is illuminated and the prevailing illuminance (Bhagavathula, Gibbons and Edwards 2015, Bullough, Donnell and Rea 2013, Donnell, Porter and Shankar 2010, Isebrands, Hallmark, Hans, McDonald, Preston and Storm 2006, Smadi, Hawkins and AldemirBektas 2011, Wortman and Lipinski 1974). Yet, studying the effect of roadway lighting on night crashes or related parameters only considers an extreme aspect of driving behavior, ignoring normal driving behaviors and critical events such as near misses. Studying the relationship between lighting design and crashes might give some insights into the effectiveness of lighting intersection designs in terms of safety. However, such a study would not reveal the full extent of the relationship between lighting design and nighttime visibility, since crashes often have multiple causal factors, making it difficult to understand the specific role of lighting design in contributing to a crash. In addition, standards based on the effect of intersection lighting design on night crashes could lead to over-lighting of intersections, which could make the intersections less safe by introducing glare to drivers and reducing visibility; over-lighting would also result in energy wastage without any substantial benefits to visibility. Using crash metrics and a consensus-based approach to intersection lighting design also does not consider the role of human visual response, nor does it account for potential interactive effects of vehicle headlamps and intersection lighting design on nighttime visibility. Existing standards also do not account for the diverse pedestrian-to-vehicle conflict points at intersections.

To recommend safe lighting standards for intersections, we should understand the relationship between intersection lighting design and nighttime visibility. Intersection lighting design refers to both the lighting configuration (part of the intersection that should be illuminated) and the illuminance at which the intersection should be maintained. To take into account human visual response, intersection lighting configurations (part of the intersection illuminated) and 
illuminance associated with them should be evaluated in terms of driver visual performance. Visual performance plays a critical role in nighttime driving as it affects the speed and accuracy of performance on the visual component of a task. Detection distance of objects (like pedestrian, targets etc.) is commonly used a measure of visual performance in nighttime roadway visibility research (Bhagavathula and Gibbons 2013, Edwards and Gibbons 2008, Hills 1975, Janoff 1993, Shinar 1985, Zwahlen and Schnell 1999). The presence of lighting and increase in lighting level greatly increases the accuracy and speed with which information can be extracted from the environment, and has been found to increase visual performance (Boyce 1973, Eloholma et al. 2006, Rea 2000, Terry and Gibbons 2015, Van Bommel and Tekelenburg 1986). Increase in visual performance of the driver at the intersection due to lighting could assist the drivers in hazard detection (pedestrians, other vehicles and animals in order to avoid collisions), and making better judgements (gap acceptance at stop - controlled intersections, yielding to traffic while making left/right turns at signalized intersections etc.). Previously, Rea et al. (2010) used photometric software to determine the Relative Visual Performance (RVP) scores for different intersection types, lighting levels and lighting layouts (continuous vs. localized). They concluded that illuminating intersections will help in increasing visibility. Further, Bullough, Donnell and Rea (2013) used a similar photometric simulation approach to understand the relationship between RVP and crash frequency at intersections in Minnesota and determined that improvement in visual performance by installing roadway lighting could lead to increased safety (lower crash frequency). These results have not yet been validated in a realistic nighttime scenarios. However, intersection lighting design has yet to be evaluated in terms of driver visual performance. For an intersection lighting design to result in increased visual performance, it is important to understand not only the effect of illuminating different parts of an intersection but 
also the effect of lighting level and possible interactive influences.

This study had two goals. The first was to evaluate different kinds of lighting configurations to determine the ones that offer the best visual performance. The second was to determine what lighting levels, perhaps specific to each lighting configuration, support the best visual performance. It was hypothesized that: (1) intersection lighting configurations would differ in visual performance measurements, since different configurations affect object contrast which, in turn, influences visual performance (Edwards and Gibbons 2008, Hills 1975); and (2) the benefit of increasing illuminance on visual performance will decrease (or plateau) at higher illuminance, consistent with the Adrian’s (Adrian 1989) Model and the RVP model (Rea and Ouellette 1991). Results from this work were intended to facilitate development of intersection lighting design standards (especially for those intersections located in isolated/rural areas) that will increase driver visual performance and consequently reduces nighttime crashes.

\section{Methods}

\section{Participants}

Twenty-four participants completed the study, and were recruited to form two age groups (younger and older), each of which was gender balanced. The younger group was comprised of participants aged 18 - 35 years $(M=30.8$ years, $S D=2.7)$, while members of the older group were all 65 years or older $(M=68.2$ years, $S D=1.6)$. These age ranges were intended to capture a wide range of driving experiences as well as a broad range of visual capabilities since human eyes undergo many physiological changes with age that result in several effects such as a decrease in visual acuity, a decrease in contrast sensitivity, an increase in dark adaptation time 
(Derefeldt et al. 1979, Evans and Ginsburg 1985, Joulan et al. 2015, Owsley et al. 1983, Salvi et al. 2006).

Participants were recruited from the Virginia Tech Transportation Institute participant database and through campus notices posted electronically, and were required to have a valid United States driver’s license. Eligible participants completed an initial screening session. In this, participants first provided written, informed consent (all experimental procedures were approved by the Virginia Tech Institutional Review Board). Participants then completed a basic visual acuity test that was administered by an Early Treatment Diabetic Retinopathy Study (ETDRS) chart with an illuminator cabinet. All participants had a minimum corrected visual acuity of at least 6/12 (20/40). Data collection took place in three sessions, on separate days, following the initial screening session. Participants were paid \$30 per hour for their participation in this study.

\section{Experimental Design}

A repeated measures experimental design was employed to investigate the effects of target location, intersection lighting configuration, and illuminance on visual performance. Visual performance was measured indirectly, using a target detection distance, while participants drove at night through a realistic roadway intersection under several conditions involving different lighting configurations and illuminances. Targets were located at multiple locations within and surrounding the intersection. This study was conducted at the intersection on the Virginia Smart Road at the Virginia Tech Transportation Institute (Figure 1). The Smart Road is a 2.2 mile long, controlled access roadway research facility built to United States highway standards. The intersection is equipped with signal lights, but they were not used for this study to eliminate the confounding effect of signal phase timing on intersection approaches. Independent 
variables and the level used in the study are summarized in Table 1, with additional details below. In a given experimental session, participants encountered one lighting configuration, all five illuminances, and all target locations within each illuminance. The remaining lighting configurations were encountered in subsequent sessions; this approach was used since changes in lighting configuration were relatively time consuming compared to changes in illuminance and target location. Presentation orders of both lighting configuration and illuminances were counterbalanced across participants to reduce potential order-related confounding effects. Target location was randomized in a given combination of lighting configuration and illuminance, with blanks (no target presentation or null condition) included as catch trials.

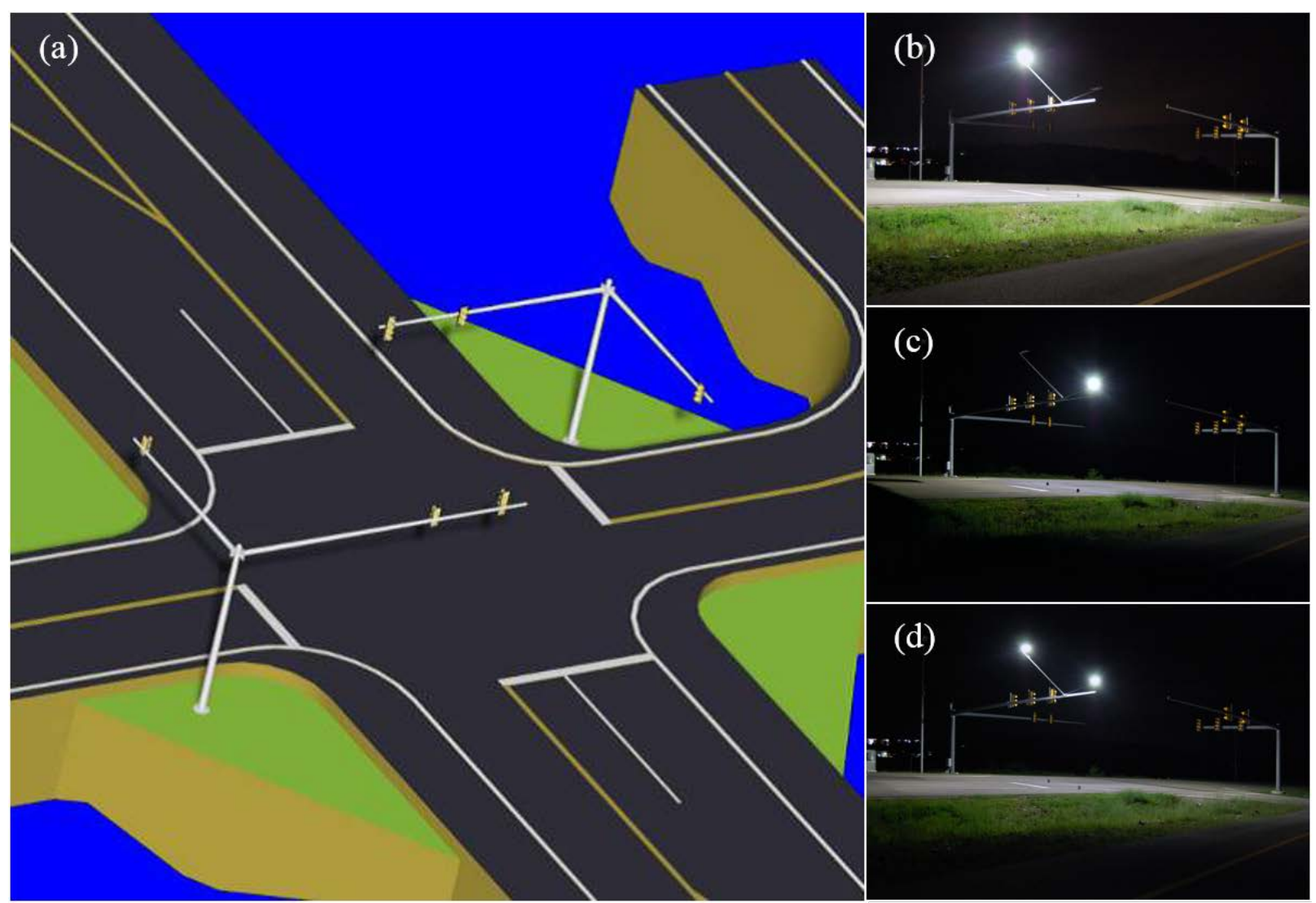

Figure 1. Diagram of the intersection on the Smart Road (a). The intersection is equipped with signal lights and lane markings associated with a typical signalized intersection. The intersection 
could also be illuminated by three configurations, which illuminated the Approach (b), the Box (c) or the Approach and the Box (d)

Table 1. Independent variables and their levels used in the experiment

\begin{tabular}{ll}
\hline Independent Variable & Levels \\
\hline Target Location & Near Right, Near Middle, Near Left, Far Right and Far \\
& Left \\
\hline Intersection Lighting Configuration & Lighted Approach, Lighted Intersection Box, Both \\
& Approach and Box Lighted \\
\hline Intersection Illuminance & 0 (no lighting), 8, 12, 16 and 21 lux
\end{tabular}

\section{Independent Variables}

\section{Target Locations}

A gray-colored wooden target, 18 x $18 \mathrm{~cm}$ (50\% reflectance), was used for the detection task, the same as targets used in earlier research on nighttime roadway visibility (Bhagavathula and Gibbons 2013, Bhagavathula et al. 2012, Gibbons et al. 2013, Gibbons et al. 2012, IESNA 2005, Janoff 1992, Janoff 1993, Mayeur et al. 2010). Five target locations were used (Figure 2), to cover several locations within/surrounding the intersection, more specifically the entrances and exits to pedestrian crosswalks. Targets were placed on the roadway (see Figure 3, Figure 4, and Figure 5Figure 7). Target locations were selected so that they contrasted against the roadway surface and not the pavement markers on the roadway. Care was taken during the initial setup to avoid shadows influencing detection. 


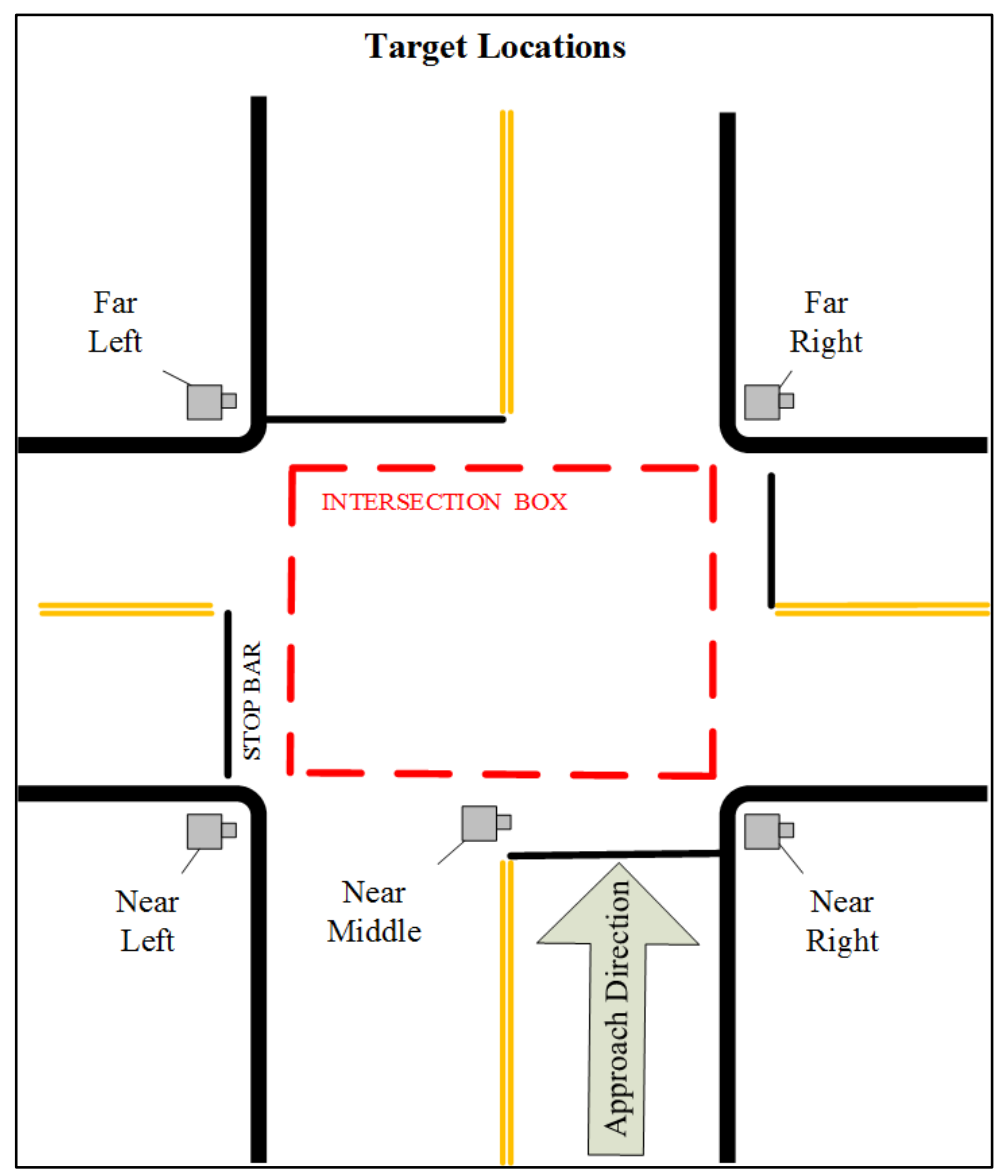

Figure 2. Overhead view of the intersection and the five target positions used. 


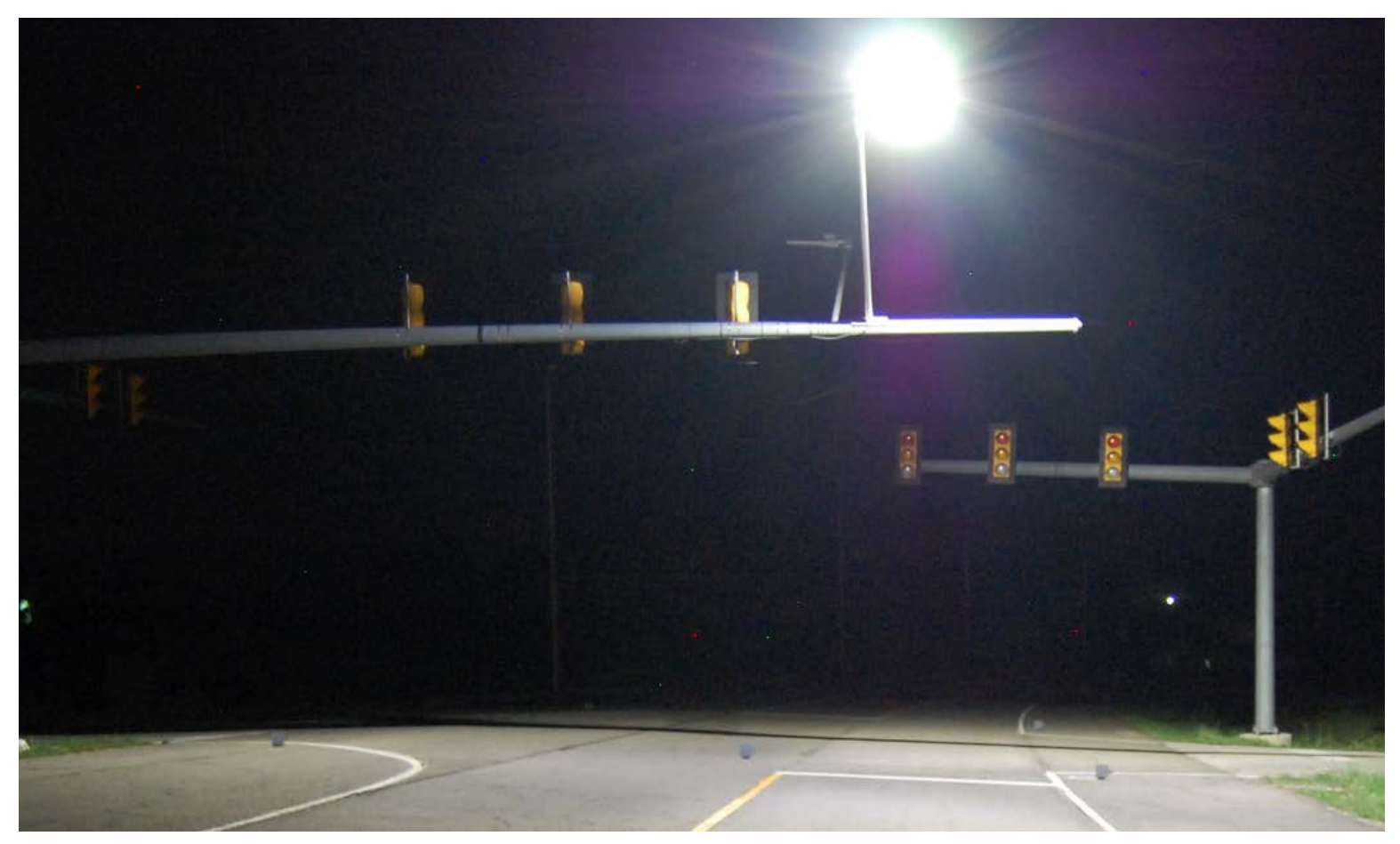

Figure 3. Targets in Approach Lighting Configuration

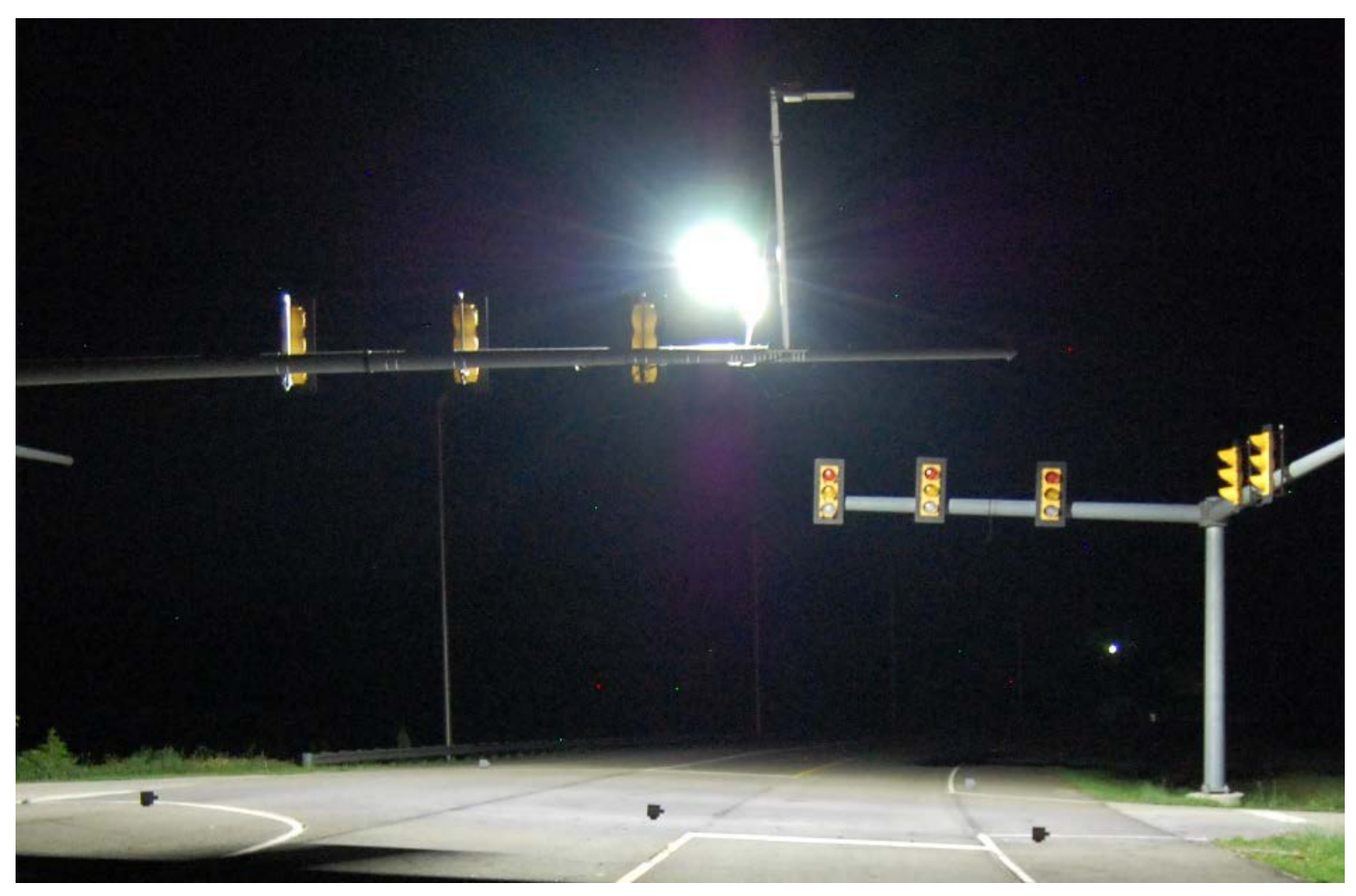

Figure 4. Targets in Box Lighting Configuration 


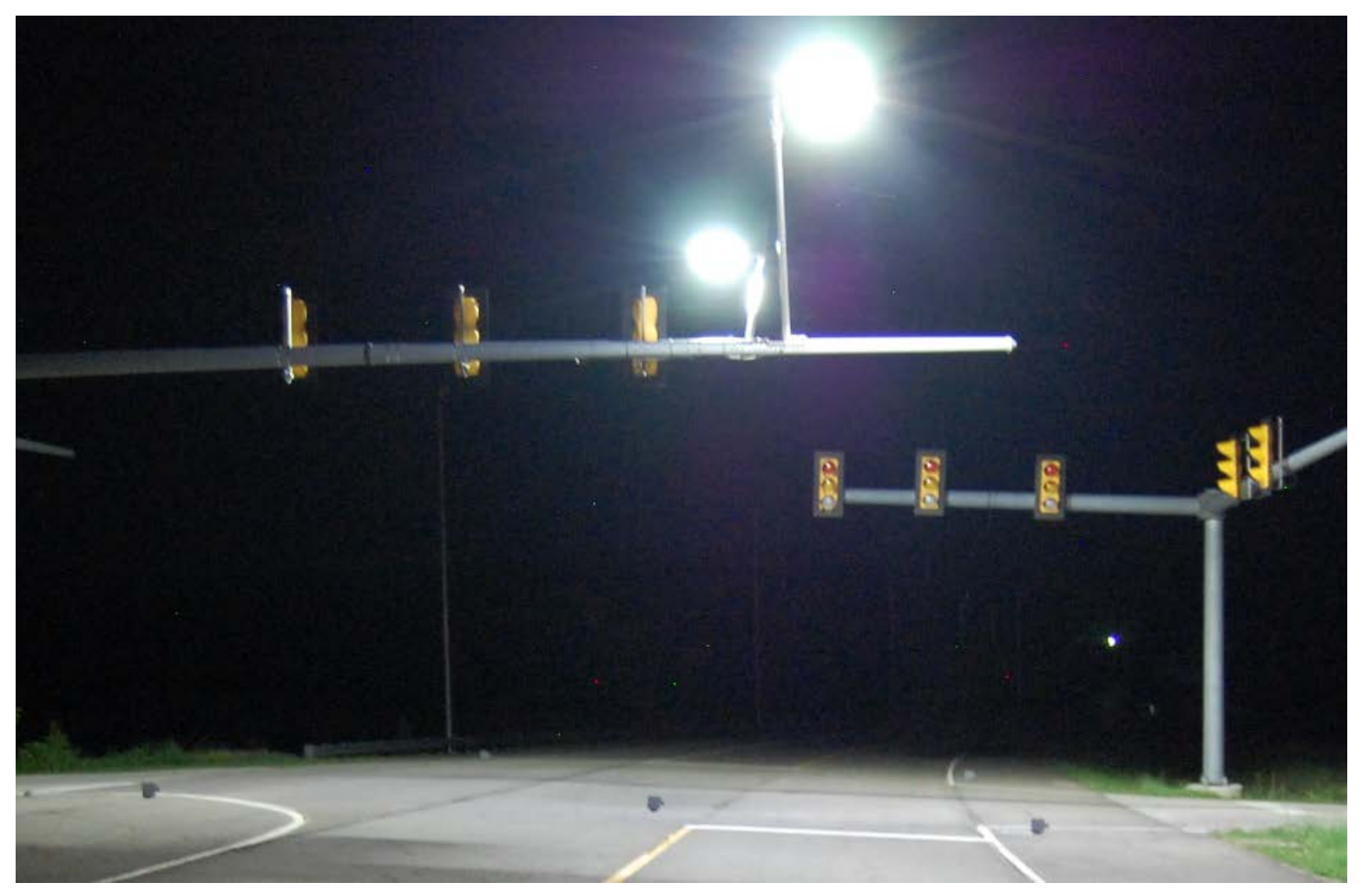

Figure 5. Targets in the Both Lighting Configuration

\section{Intersection Lighting Configurations}

Three different intersection lighting configurations were developed (Figure 2), and classified based on the part of the intersection that was illuminated. In the first configuration (Approach), the approach to the intersection was primarily illuminated (Figure 1b and Figure 6a). In the second configuration (Box), the intersection box was illuminated (Figure 1c and Figure 6b). The third configuration (Approach and Box) had both the approach and box of the intersection illuminated (Figure 1d and Figure 6c). As well as assessing different lighting configurations, these alternatives also allowed for testing the effects of the two different kinds of contrast (positive and negative) of the target located at the near right and near middle target locations on the visual performance of drivers. Specifically, the Approach configuration rendered these targets in positive contrast, since the face of the target was brighter than the 
background (Figure 7a). The Box configuration rendered these targets in negative contrast, since the background was brighter than the face of the target and it appeared in silhouette (Figure 7b). In the Approach and Box configuration, target contrast will depend on the illuminance. In addition to the contrasts in which the targets were rendered, the other reason for selecting these three lighting configurations is that the goal was to get a symmetrical lighting design at the intersection so that the results could be generalized for all approaches. For example, illuminating the area after the intersection, it would render all the objects in negative contrast for a driver approaching in one direction. However, for a vehicle approaching from the opposite direction, the targets would be rendered in positive contrast. 


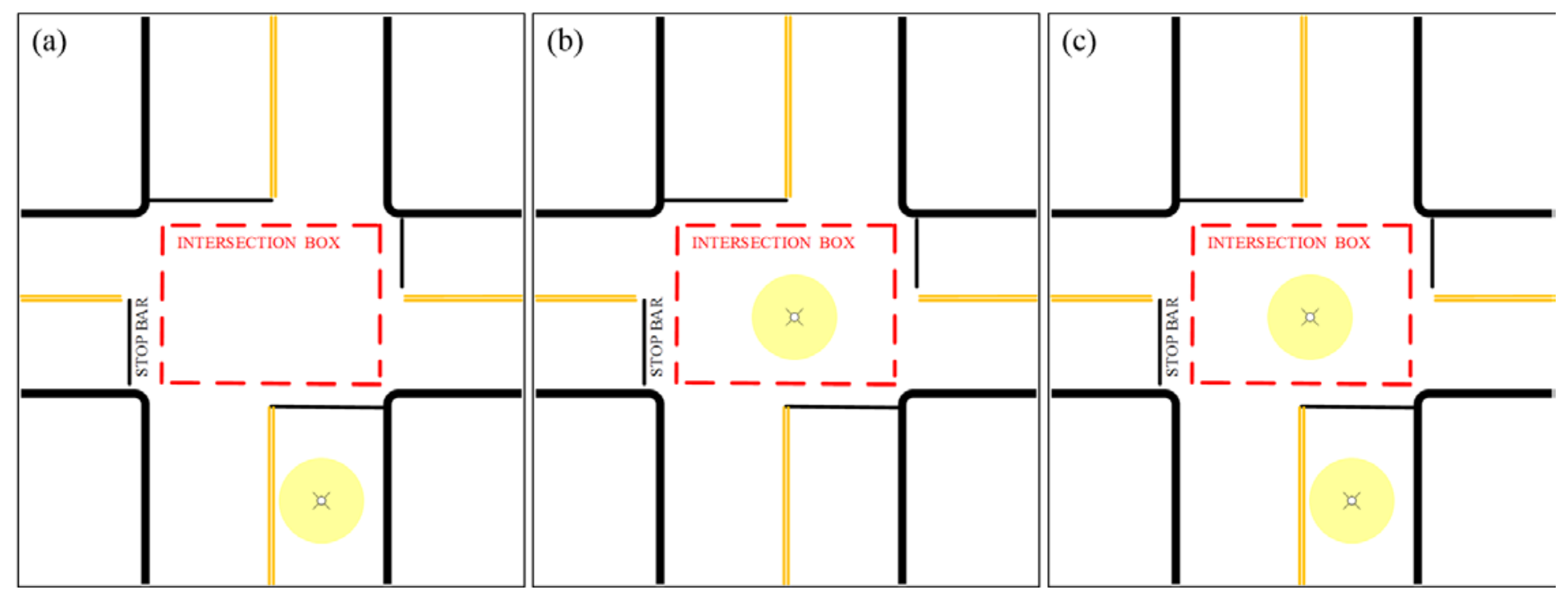

Figure 6. Illustrations of the three intersection lighting configurations: (a) Intersection approach is illuminated, (b) Intersection box is illuminated. (c) Both the box and approach are illuminated. 


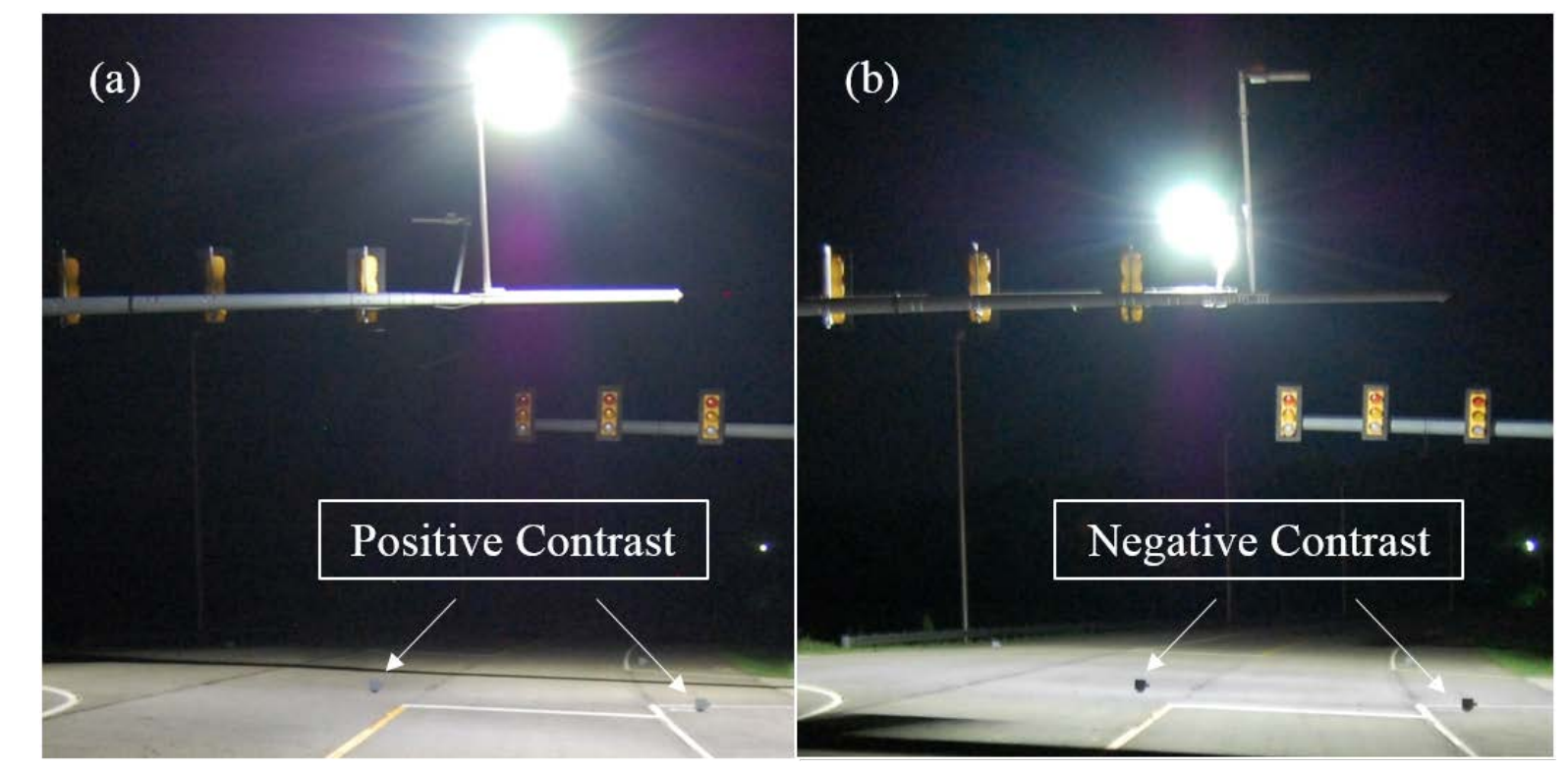

Figure 7. Near right and near middle target locations in positive and negative contrast in the Approach (a) and Box (b) lighting configurations. Targets were placed on the roadway. 


\section{Intersection Illuminance}

Two 4000 Kelvin light emitting diode (LED) luminaires were used for illuminating the intersection, and were mounted at height of $8.5 \mathrm{~m}$. Luminaires used for illuminating the approach and the box were, respectively, type II and type V, and which had different light distribution patterns. Types II luminaires are used for illuminating roadways; these are typically mounted near the edge of the roadway and have an elliptical light distribution pattern. Type V luminaires are typically mounted in the center of a four-way intersection and have a circular light distributions with the same light intensity in all lateral directions (Murdoch 2003).

Five different illuminances were used, and intended to span a range of recommended values. The specific levels were 0 (no lighting), 8, 12, 16, and 21 lux, and were the horizontal illuminance measured at the near right target location on the intersection and not the mean of value of multiple points or measures. The two lowest light levels (8 and 12 lux) are also the IESNA RP-8 recommended average illuminance at low nighttime pedestrian volume locations, such as those at rural/sub-urban areas (IESNA 2005). The 16 and 21 lux levels are the IESNA RP-8 recommended average illuminance for high and medium pedestrian conflict areas at sub-urban and urban locations (IESNA 2005). Since it was impossible to maintain the same illuminance at every target location under different lighting configurations, only the near right target location was selected to match illuminance across the lighting configurations. The horizontal illuminance for each target location and the mean horizontal illuminance of the intersection (mean intersection illuminance) are shown in Table 2 for each lighting configuration and illuminance. Mean horizontal illuminance was the average horizontal roadway surface illuminance of the area enclosed by the stop-bars at the intersection. 
Table 2. Horizontal illuminance at the target locations and mean intersection illuminance used in the study for each lighting configuration and illuminance.

\begin{tabular}{|c|c|c|c|c|c|c|}
\hline \multicolumn{7}{|c|}{ Approach Lighting Configuration } \\
\hline Illuminance (lux) & $\begin{array}{c}\text { Near } \\
\text { Right }\end{array}$ & $\begin{array}{c}\text { Near } \\
\text { Middle }\end{array}$ & $\begin{array}{c}\text { Near } \\
\text { Left }\end{array}$ & $\begin{array}{c}\text { Far } \\
\text { Right }\end{array}$ & $\begin{array}{c}\text { Far } \\
\text { Left }\end{array}$ & $\begin{array}{c}\text { Mean } \\
\text { Intersection } \\
\text { Illuminance }\end{array}$ \\
\hline $\mathbf{8}$ & 8.8 & 6.3 & 4.4 & 0.9 & 0.2 & 5 \\
\hline $\mathbf{1 2}$ & 12.6 & 9.4 & 6.4 & 1.5 & 0.2 & 7 \\
\hline $\mathbf{1 6}$ & 17.1 & 12.5 & 8.6 & 1.8 & 0.3 & 9 \\
\hline $\mathbf{2 1}$ & 22.5 & 16.3 & 11.6 & 2.4 & 0.4 & 12 \\
\hline \multicolumn{7}{|c|}{ Box Lighting Configuration } \\
\hline $\mathbf{8}$ & 9.0 & 9.6 & 8.3 & 2.5 & 2.1 & 7 \\
\hline $\mathbf{1 2}$ & 13.2 & 14.0 & 12.2 & 3.3 & 3.1 & 10 \\
\hline $\mathbf{1 6}$ & 17.1 & 18.5 & 16.1 & 4.7 & 4.0 & 13 \\
\hline $\mathbf{2 1}$ & 22.1 & 24.4 & 21.4 & 6.4 & 5.8 & 18 \\
\hline $\mathbf{7}$ & Both Lighting Configuration & \\
\hline $\mathbf{8}$ & 8.7 & 7.2 & 5.8 & 1.6 & 1.0 & 5 \\
\hline $\mathbf{1 2}$ & 13.3 & 11.2 & 8.9 & 2.4 & 1.4 & 8 \\
\hline $\mathbf{1 6}$ & 17.0 & 15.1 & 12.8 & 3.2 & 2.2 & 11 \\
\hline $\mathbf{2 1}$ & 22.4 & 19.7 & 14.8 & 3.6 & 2.4 & 13 \\
\hline
\end{tabular}

\section{Experimental Procedures and Dependent Measure}

Participants were scheduled to arrive 15 minutes prior to the start of data collection in each experimental session. Sessions were conducted at night (after civil twilight) and only in clear weather conditions (no rain, snow, fog, etc.). Two participants were scheduled each experimental session for efficiency (see below). In the first experimental session, after arrival, participants initialled the informed consent again, reviewed the activities listed for the session, and were shown sample images of a target they might encounter during the study. A definition of a detection task was provided, along with an example of how they should respond when they see a target. In the subsequent two experimental sessions, participants were given the choice to review the experimental protocol prior to starting the session. At all times during the driving portion of the study, an experimenter was in the vehicle with the participant. The experimenter was seated in the rear passenger seat of the experimental vehicle. 
Two vehicles were used (1999 and 2000 Ford Explorers), which were instrumented with data acquisition systems (DAS) connected to the vehicles’ controller area network (CAN) and onboard camera systems. The DAS collected kinematic data from the vehicle’s CAN system, including vehicle speed, differential Global Positioning System (DGPS) coordinates, four video images (driver's face, forward roadway, left side of roadway, and right side of roadway), audio from the driver, manual button presses, and other input from an in-vehicle experimenter. Low beam headlamps were used during study and were aimed before each experimental session.

Once in the vehicle, participants were shown the locations of the vehicle's seat adjustment buttons, steering height adjustment buttons, headlamp switch, windshield wiper switch, etc. Participants were then given several minutes to familiarize themselves with the vehicle. Once the participants indicated they were comfortable and all their questions/concerns were answered, they were asked to drive the vehicle onto the Smart Road. Before entering the Smart Road, participants were informed that the speed limit for the study was $56 \mathrm{~km} / \mathrm{h}$ (35 mi/h). Participants were also informed about where to stop and turn. After entering the Smart Road, participants completed two practice "laps” (see Figure 8), in which they practiced the target detection task (under the no lighting configuration). In each lap, the first participant would approach the intersection while the second would wait at the start point. After the first participant completed the approach they were instructed to wait for the second vehicle at the end point with all lights turned off. The second participant then began their approach and arrived at the end point. From there, both participated drove back to the start point and were instructed to be ready to begin the next lap. 


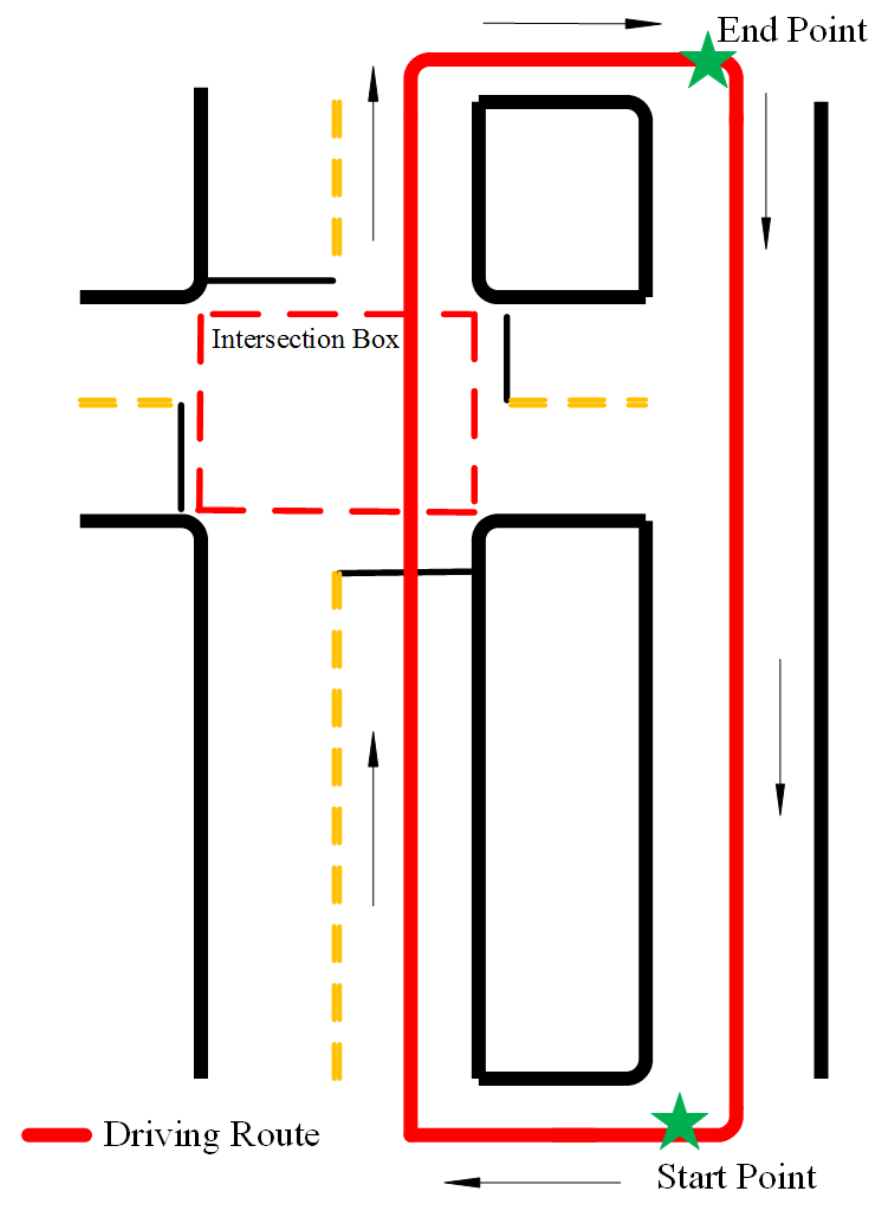

Figure 8. Overhead view of the Smart Road intersection. The thick red rectangle shows the start and end points of each lap. Participants completed 30 laps per session.

Subsequently, participants approached the intersection six times for each illuminance condition (five target locations and one catch trial), always driving straight through the intersection. During each approach, participants were asked to verbally indicate when they saw a target, by saying the word "target" out loud. Response time was recorded (by the in-vehicle experimenter) by a button press in the data stream being recorded by the DAS. At the time the vehicle passed the target, the experimenter pressed another button. These button presses were used as indices in the data stream recorded by the DAS. These indices were used to determine the exact point of detection by the participant by watching and listening to the audio-video stream recorded by the DAS. The 
DGPS coordinates of each target were pre-recorded. The DGPS coordinates at the point of detection between the car and the target were used to calculate the detection distance, which was used as the dependent measure. The accuracy of the detection distances calculated using the DGPS system was about $0.2 \mathrm{~m}$ (0.66 ft.). The target was moved (or removed for catch trials) between laps, at a time when the intersection was not visible to participants (i.e., when they were heading to the start point from the end point (see Figure 8). After target presentation was completed for one illuminance condition, an additional six approaches were completed for each of the remaining illuminance conditions. These same procedures were repeated in the second and third sessions for the two other lighting configurations.

\section{Data Reduction}

The data reduction process helped eliminate the time delay due to the button press from the experimenter. During data reduction, the data stream from the vehicle was processed by adjusting the time stamp of detection to instant when the participant said "target" from the experimenter button press. Such post hoc processing was performed using an in-house data analysis and reduction tool which synchronized all the data (audio, video, kinematic, GPS etc.) collected from the vehicle’s data acquisition system and presented it to the researchers. By capturing the coordinates at the instant the participant detected (or says when he/she detected the target) the time lag due to experimenter was eliminated. The experimenter button press just served as an index in the data stream to facilitate data reduction.

\section{Analyses}

A linear mixed model (LMM) analysis was used to assess the (fixed) effects of target location, lighting configuration, and illuminance on detection distance. Age was included as a 
blocking factor. Based on preliminary analysis of LMM residuals, the detection distances square root transformed because the model residuals using untransformed values were not normally distributed. The square root transformation allowed the model residuals to be normally distributed, which satisfied the parametric model assumptions.. The level of significance was $p$ $<0.05$ for all statistical tests. Effect sizes were determined and reported using partial eta-squared $\left(\eta_{\mathrm{p}}{ }^{2}\right)$. Where relevant, post hoc analyses (pairwise comparisons) were performed using Tukey's honest significant difference (HSD) for main effects and simple effects testing for interaction effects. Back transformed means and standard errors are also reported.

To investigate if targets were detected from a "safe" distance under each combination of lighting configuration and illuminance, mean detection distances across the five target were compared to the stopping sight distance ((AASHTO 2011). Stopping sight distance is the length of the roadway required for a vehicle travelling at the "design speed" (here, $56 \mathrm{~km} / \mathrm{h}$ or $35 \mathrm{mph}$ ) to come to a stop, and is the distance travelled by the vehicle from the time a driver sees an object to the vehicle coming to a complete stop (sum of distance travelled during brake reaction time and braking distance). For the purpose of recommending the safe stopping distance, a brake reaction time of 2.5 seconds and deceleration rate of $3.4 \mathrm{~m} / \mathrm{s}^{2}$ is assumed by AASHTO. Based on AASHTO (2001), and given the $56 \mathrm{~km} / \mathrm{h}$ (35 mph) driving speed used in the current study, if the mean detection distance was greater than 76.2 meters ( $250 \mathrm{ft}$.) for a given target location, then the driver would have had enough distance to stop safely after detection. Thus, 76.2 meters was used as a basis for assessing target detection distances. 


\section{Results}

Overall there were 1872 presentations, including both targets and blanks (no target presentation or “catch” trials). Of these 1872 target presentations, there were 312 presentations for each target location (1560 total target presentations) and 312 blank presentations. Out of 1560 target presentation across all participants, there were three false detections. False detections are cases when the participants reported a target when a target was not presented (catch trial). This small number was not unexpected, as the experimental designed was not a forced-choice paradigm. Rather, participants could, in the majority of cases, eventually see the target since it was always present; it was just a matter of the distance at which they could actually see the target. This distance was affected by how well a certain lighting design and illuminance affected targets’ visibility. False detections were not analysed, and were excluded from the LMM analysis. A total of 130 targets were missed and were also excluded from the LMM analysis. The percentage of misses depended on target location and lighting configuration. Participants missed higher percentage of targets in the Approach lighting configuration (14.2\%) than in the Box (3.7\%) or Both (6.3\%) configurations. In the Approach configuration, near left and far left target locations had the highest percentages of misses (Figure 9). 

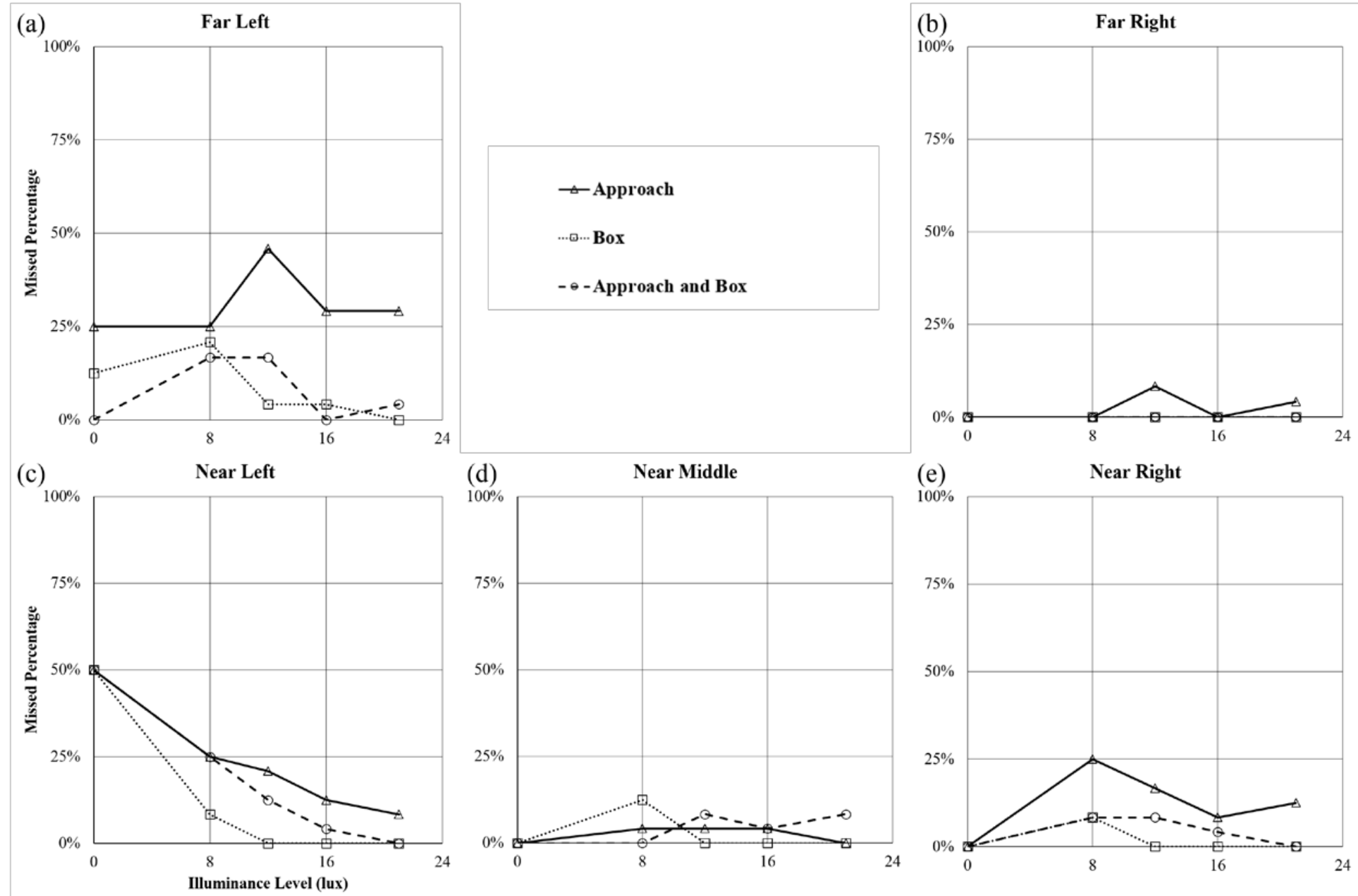

Figure 9. Percentage of missed targets by location and lighting configuration. 
All LMM results are summarized in Table 3. All main effects were significant, along with several two-way interactions, and the three-way interaction of target location, lighting configuration and illuminance. Subsequent subsections provide additional details on the results regarding age and lighting configuration, followed by the noted three-way interaction effect.

Table 3. Statistical results from linear mixed model analysis of detection distance. Significant effects are highlighted using bold text.

\begin{tabular}{|l|c|c|}
\hline Effect & $\begin{array}{c}\text { Effect Size } \\
\left(\boldsymbol{\eta}_{\mathbf{p}}{ }^{2}\right)\end{array}$ & $\boldsymbol{p}$ value \\
\hline Age (A) & $\mathbf{0 . 1 5}$ & $\mathbf{0 . 0 3 2 4}$ \\
\hline Target Location (TL) & $\mathbf{0 . 2 5}$ & $<.0001$ \\
\hline $\begin{array}{l}\text { Lighting Configuration } \\
\text { (LC) }\end{array}$ & $\mathbf{0 . 1 6}$ & $<.0001$ \\
\hline Illuminance Level (IL) & $\mathbf{0 . 0 6}$ & $<.0001$ \\
\hline A x LC & $\mathbf{0 . 0 0}$ & $\mathbf{0 . 0 4 1 7}$ \\
\hline A x IL & 0.00 & 0.6698 \\
\hline A x TL & 0.00 & 0.4353 \\
\hline LC X IL & $\mathbf{0 . 0 5}$ & $<.0001$ \\
\hline TL x LC & $\mathbf{0 . 1 2}$ & $<.0001$ \\
\hline TL X IL & 0.01 & 0.3504 \\
\hline A X LC x IL & 0.01 & 0.1727 \\
\hline A x TL x LC & 0.00 & 0.675 \\
\hline A x TL x IL & 0.01 & 0.2071 \\
\hline TL x LC x IL & $\mathbf{0 . 0 3}$ & $\mathbf{0 . 0 2 2 1}$ \\
\hline A x TL x LC x IL & 0.01 & 0.9906 \\
\hline
\end{tabular}

\section{Interactive Effect of Age and Lighting Configuration}

Detection distances were longer for the younger age group in all three lighting configurations, though the difference between age groups was inconsistent across the three configurations (Figure 10). Simple effects tests indicated that differences between groups were significant only for the Both and Box configurations, with younger participants having 32 and $\sim 27 \%$ longer distances in these configurations, respectively. Simple effects of lighting 
configurations were also significant in both age groups, and in which detection distances were longest in the Box configuration and shortest in the Approach configuration.

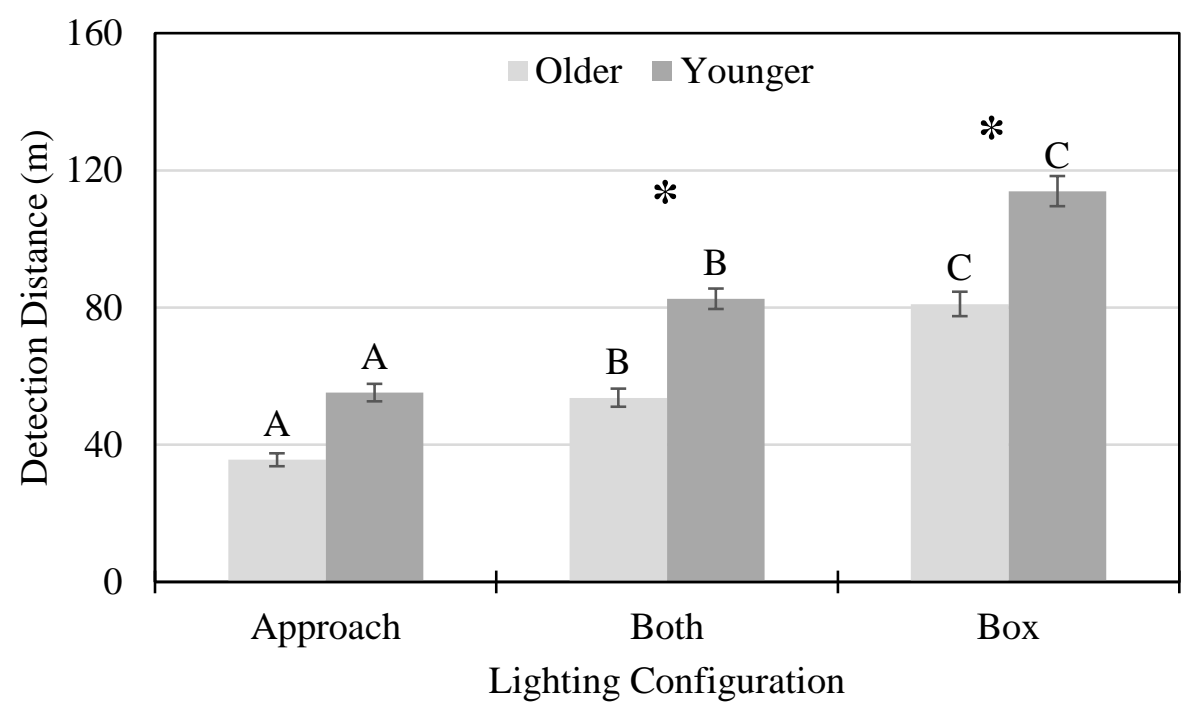

Figure 10. Effects of age and lighting configuration on detection distance. Values are means of detection distances and error bars indicate standard errors. Uppercase letter represent post-hoc groups between lighting configurations in each age group, and the symbol * indicates a significant difference between age groups in each lighting condition.

\section{Interactive Effect of Target Location, Lighting Configuration and Illuminance}

The combined effects of target location, lighting configuration, and illuminance on detection distance are summarized in. Two analysis approaches were used to further assess this three-way interaction effect, and with an emphasis on two aspects that were considered most practically relevant. The first examined the effect of lighting configuration on detection distance at each illuminance for each target location, which focused on the differences between configurations and the consistency of these differences across illuminance and target locations. The second examined the effect of illuminance on detection distance at each lighting 
configuration for each target location, and which was used to assess plateaus that were evident in detection distances with increasing illuminance.

\section{Effect of Lighting Configuration}

Overall, detection distances were longest in the Box lighting configuration and shortest in the Approach configuration $\left(M_{\text {Box }}=108.04 \mathrm{~m} ; \mathrm{M}_{\text {Both }}=75.95 \mathrm{~m} ; M_{\text {Approach }}=52.32 \mathrm{~m}\right)$; this pattern of results was consistent for each of the illuminance conditions. From simple effects testing, the effect of lighting configuration was significant at every illuminance for the near right, near middle, near left, and far left target locations. For the remaining (far right) target location, differences in detection distances between lighting configurations were only significant at the 12 lux illuminance. 

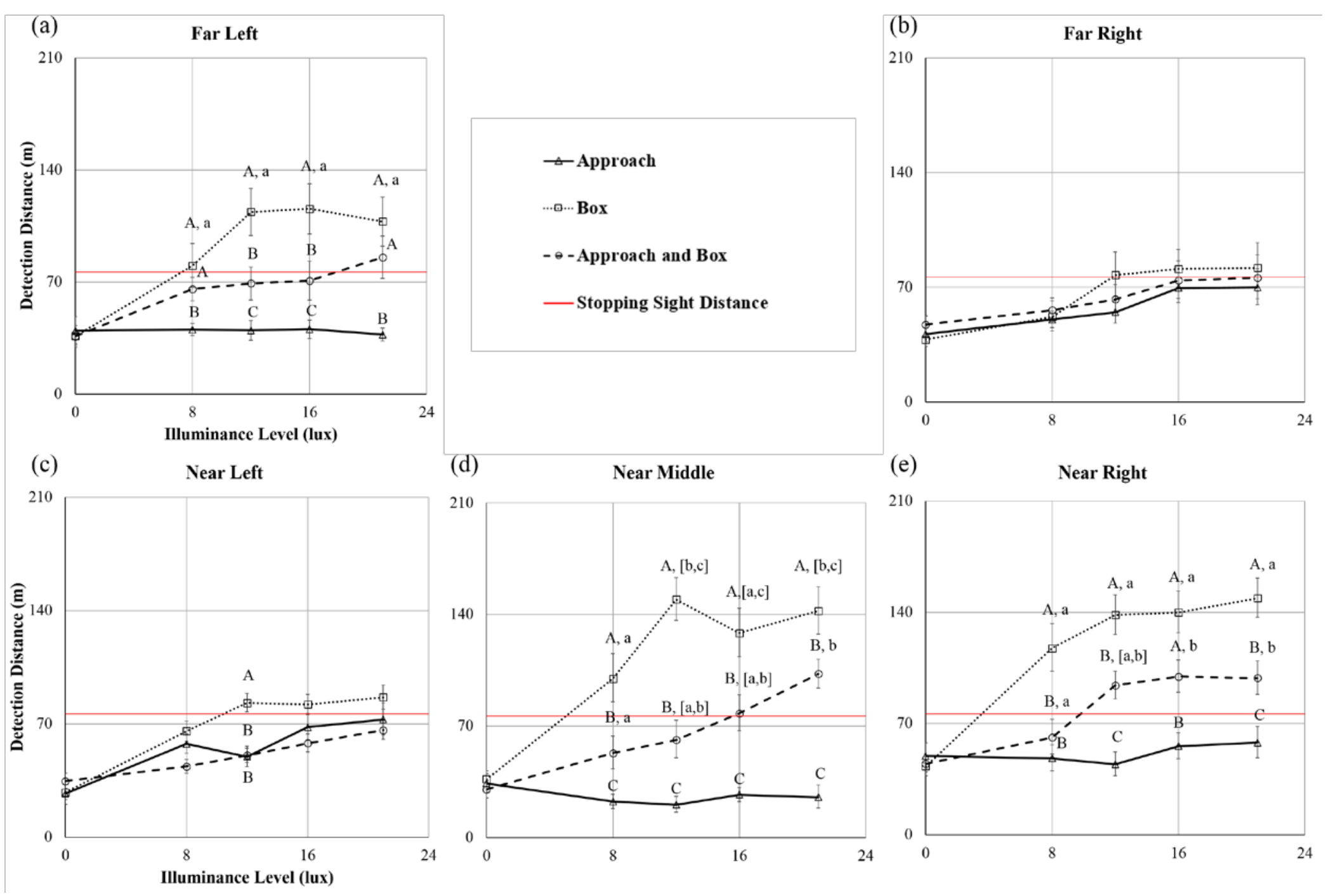
Figure 11. Interactive effects of lighting configuration and illuminance at each target location. Values are means of detection distances and error bars reflect standard errors. Uppercase letters indicate post-hoc groupings (from paired comparisons) between lighting configurations at a given illuminance level, while lower case letters indicate such groupings between illuminances for a given lighting configuration. Horizontal red lines indicate the stopping sight distance at $56 \mathrm{~km} / \mathrm{h}(35 \mathrm{mi} / \mathrm{h})$. 
Assessment of the post-hoc pairwise comparisons revealed the existence of significant differences in detection distances between the lighting configurations at every illuminance and target location. For near right (Figure 11e), near middle (Figure 11d) and far left (Figure 11a) target locations, detection distances in the Box lighting configuration were significantly longer than those in the Both and Approach lighting configurations at every tested illuminance greater than 0 lux. For the near left target location (Figure 11c), pairwise comparisons were significant between the three lighting configurations only at the 12 lux illuminance. No pairwise comparisons between lighting configurations were significant for the far right target location (Figure 11b). At every illuminance, the Box lighting configuration had longer detection distances than the Both configuration by approximately 25 to $50 \%$ depending on the illuminance and the Approach configuration by approximately 50 to $60 \%$ depending on the illuminance.

To isolate the effect of the intersection lighting configuration on detection distances, the mean detection distance in the no lighting condition (headlamp only) were subtracted from the mean detection distances at each illuminance level in all the three lighting configurations. The results showed that the mean detection distances of all target under the Box lighting configuration were higher than Approach and Both lighting configurations at all illuminance levels (see Figure 12). In Box and Both lighting configurations, the mean detection distance with the roadway lighting increased with increasing illuminance. The increase in the mean detection distance with increase in the illuminance were the highest in the Box lighting configuration (ranging from $137 \%$ to $219 \%$ of the mean detection distance in the headlamps only condition) followed by the Both lighting condition (26\% to $101 \%$ of the mean detection distance in the headlamps only condition). In the Approach lighting configuration, the increase in the detection distance was the 
lowest (ranging from $-14 \%$ to $21 \%$ of the mean detection distance in the headlamps only condition).

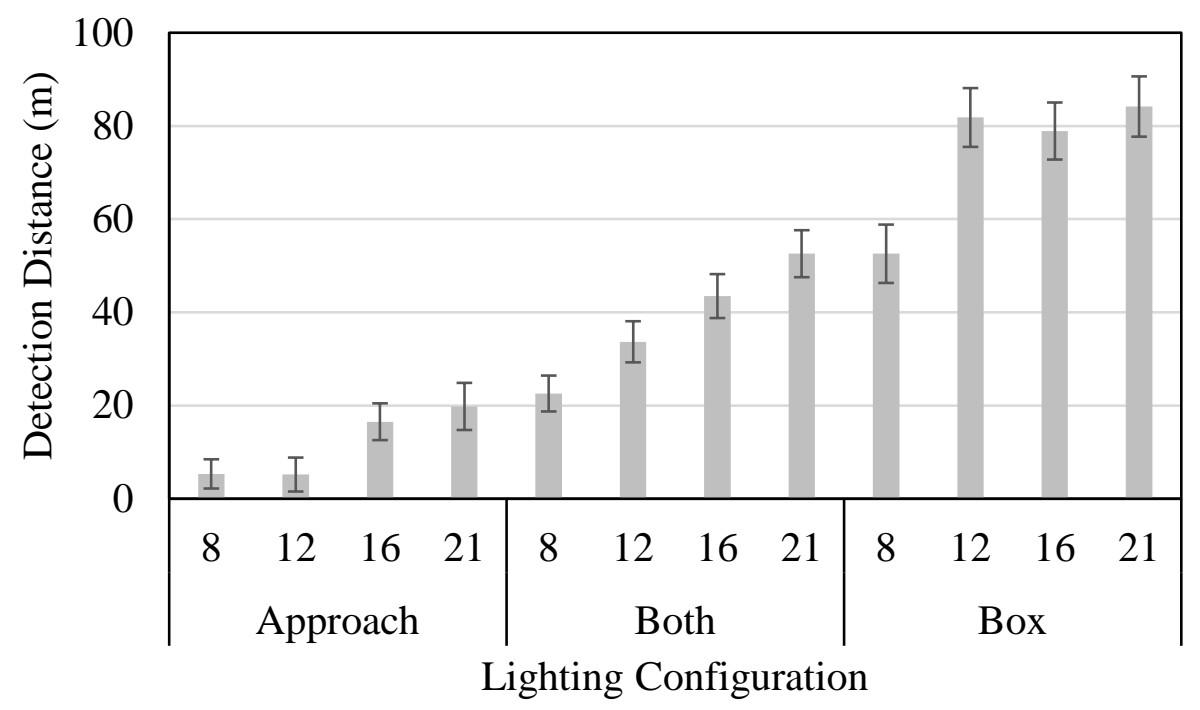

Figure 12. Effect of lighting configuration and illuminance without taking the headlamps of the vehicle into consideration. Values are means of detection distances in each lighting configuration and illuminance with the mean detection distance in the no-lighting condition subtracted. Error bars represent standard errors.

\section{Effect of Illuminance}

There appeared to be an illuminance at which detection distance plateaued within each lighting configuration and for every target location (see Figure 11). Simple effects testing revealed that illuminance had a significant effect on detection distance for near right, near middle, and far left target locations in the Box and Both lighting configurations. At the near left target location, illuminance had a significant effect on detection distances in all three lighting configurations. At the far right target location, illuminance had a significant effect on detection distances in the Approach and Box lighting configurations. 
Assessment of post-hoc pairwise comparisons confirmed the existence of plateaus in detection distance for several target locations in the different lighting configurations. These plateaus occurred for all targets (in the range of the illuminances evaluated) excepting the far right, though inconsistently across lighting configurations (Table 4). Four of the five target locations (except far right) had such a plateau in the Box lighting configuration. In the Approach and Both lighting configurations, only two and one target locations showed the detection distance plateau, respectively. Furthermore, the mean detection distance at which the plateau occurred was significantly higher and occurred at a lower illuminance in the Box vs. the Approach and Box lighting configurations (Figure 11).

Table 4. Illuminance at which detection distance plateaus occurred or was not evident (based on paired comparisons) for each combination of target location and lighting configurations at each.

\begin{tabular}{|l|c|c|c|}
\hline \multirow{2}{*}{$\begin{array}{l}\text { Target } \\
\text { Location }\end{array}$} & \multicolumn{3}{|c|}{ Lighting Configuration } \\
\cline { 2 - 4 } Near Right & No Plateau & 8 lux & 12 lux \\
\hline Near Middle & No Plateau & 12 lux & 12 lux \\
\hline Near Left & 16 lux & 12 lux & No Plateau \\
\hline Far Right & No Plateau & No Plateau & No Plateau \\
\hline Far Left & No Plateau & 8 lux & No Plateau \\
\hline
\end{tabular}

\section{Comparisons of Mean Detection Distance to Safe Stopping Distance}

Overall, $48.3 \%$ of the target locations were detected from a safe distance (based on the value of $76.2 \mathrm{~m}$ (250 ft.) as described earlier). In the Box lighting configuration, 90\% of target locations were detected from a safe distance, while only 45 and 10\% were detected at a safe distance in the Approach and Both configurations, respectively.

In the Box configuration, near right, near middle and far left target locations had mean detection distances greater than the stopping sight distance for all levels of illuminance except the no lighting condition (Figure 11). For the remaining targets in this same lighting configuration (near 
left and far right), the mean detection distance was greater than the stopping sight distance for three illuminances: 12, 16 and 21 lux. In the Approach configuration, only the near left and far right target locations at the highest illuminance (21 lux) had mean detection distances greater than the stopping sight distance (Figure 11). In the Both lighting configuration, the following target locations had mean detection distances greater than the stopping sight distance (Figure 11): near right (12, 16 and 21 lux), near middle (16 and 21 lux), far right (16 and 21 lux) and far left (16 and 21 lux).

\section{Discussion}

The goals of this study were to determine whether a driver's visual performance, measured using a target detection task, differs between three intersection lighting configurations and to identify the illuminance that offers the best visual performance within each intersection lighting configuration. Three major findings were evident. First, there was a significant difference in visual performance between the three lighting configurations with the Box lighting configuration having the longest and the Approach lighting configuration having the shortest detection distances $\left(M_{\text {Box }}=108.0 \mathrm{~m} ; M_{\text {Both }}=75.9 \mathrm{~m} ; M_{\text {Approach }}=52.3 \mathrm{~m}\right)$. Second, the effect of illuminance on visual performance within each lighting configuration was not consistent, but rather was dependent on target location. Third, age-related differences in visual performance measurements were consistent across the conditions investigated, with the younger participants having better visual performance (longer detection distances) than older participants.

Regarding the effects of lighting configuration, three converging lines of evidence indicate that the Box lighting configuration yielded superior visual performance. First, longer detection distances were found with Box lighting than either the Approach or Both configurations, and this 
was found at every illuminance and target location. Second, 90\% of target locations were detected from a safe distance under the Box lighting configuration, compared to 45 and $10 \%$ in the Box and Approach configurations, respectively. Third, participants missed (failed to detect) fewer targets in the Box lighting configuration (3.7\%) than in the Approach (14.2\%) and Both (6.3\%) configurations.

Superior visual performance in the Box lighting configuration is likely a result of the contrast in which the targets locations were rendered. Generally, the visibility of objects at nighttime depends on their contrast with the relevant background (Edwards and Gibbons 2008, Pretto and Chatziastros 2006). With respect to intersection, target contrasts are affected by the lighting configuration (part of the intersection illuminated) and the headlamps of the vehicle. Those target locations rendered in negative contrast in the Box lighting configuration (i.e., near right and near middle) had significantly longer detection distances than when the same target locations were rendered in positive contrast in the Approach lighting configuration, and this difference was found at every illuminance greater than 0 lux. This finding implies that negative contrast on targets results in better nighttime visual performance than positive contrast. Such a result is consistent with work reported by Aulhorn (1964) and Hills (1975), who showed that objects in negative contrast (Weber contrast) were detected faster and from farther than those in positive contrast. However, it is important to note that Aulhorn (1964) and Hills (1975) used positive and negative contrasts of the same magnitude, while in this study the magnitude of the contrasts in which the targets were rendered in was not controlled. Furthermore, a model of visibility suggested by Adrian (1989) contains an adjustment factor for contrast polarity, which makes targets in negative contrast twice as detectable as those in positive contrast. Comparing of mean detection distances here in the Approach vs. Box lighting configurations for the younger and 
older participants showed that negatively contrasted targets had detection distances that were 2.8 times higher than positively contrasted targets (range $=1.7$ to 4 times depending on age and light level), which is higher than the value suggested by Adrian. However, a more detailed photometric analysis, which is beyond the scope of this paper, is required accurately understand the influence of contrast polarity on detection. The result of small target being detected easily in negative contrast than in positive contrast has also been reported by Fotios et al. (2016), who studied the detection of small obstacles by cyclists with bicycle mounted lamps and roadway lighting. The contrast polarity (negative/positive) of the near right and near middle targets in the Both lighting configuration depended on the illuminance, since both the area in front of and behind the target locations were illuminated, and a photometric analysis is required in the future to accurately determine the contrast on these targets.

Targets can also undergo a change in contrast polarity (negative to positive or vice versa) from the point of view of driver in a moving vehicle. For example, near right and near middle target locations were originally rendered in negative contrast in the Box lighting configuration, but slowly transitioned into positive contrast as the vehicle moved closer to the target and the headlamps illuminate the face of the target to be brighter than the background. Headlamps, though, only generate a substantial influence at distances less than 100 meters to the target (Edwards and Gibbons 2008). Our results indicated that the negatively contrasted targets in the Box lighting configuration had mean detection distances $>100$ meters even at the 8 lux illuminance, whereas the positively contrasted targets in the Approach lighting configuration had mean detection distances well under 100 meters at the highest illuminance of 21 lux. The shorter mean detection distances in the Approach lighting configuration also suggest that the magnitude of positive contrast in which the near right and middle target locations were rendered is not 
sufficient to be detected by participants and that additional luminance from the headlamps is required to further increase the contrast and facilitate detection. The mean detection distances of the same targets in the Box lighting configuration, however, were typically detected beyond the range of headlamps.

Targets rendered in the same contrast polarity also exhibited different levels of visual performance across the tested lighting configurations. For example, the far left target location was rendered in positive contrast in all three lighting configurations, yet detection distances in the Box lighting configuration were higher than the other two configurations. The differences between lighting configurations could be attributed to the far left target being rendered in a higher positive contrast in the Box lighting configuration than in the other two lighting configurations. Further, the far left target had a higher mean detection distance than the far right target in the Box lighting configuration, even though both targets were rendered in positive contrast. On further examination of target locations, this phenomenon could be due to influence of background luminance at these target locations, as viewed by the approaching driver. Specifically, the far right target seemed to have a higher background luminance, being contrasted against the pavement, whereas the far left target was contrasted against the darker region beyond the pavement. The darker background, and associated lower background luminance ( $\mathrm{L}_{\mathrm{bg}}=0.05$ $\mathrm{cd} / \mathrm{m}^{2}$ ), likely caused the far left target to have a higher contrast compared to the far right target location where the background luminance was higher $\left(\mathrm{L}_{b g}=0.11 \mathrm{~cd} / \mathrm{m}^{2}\right)$ (Figure 13). 


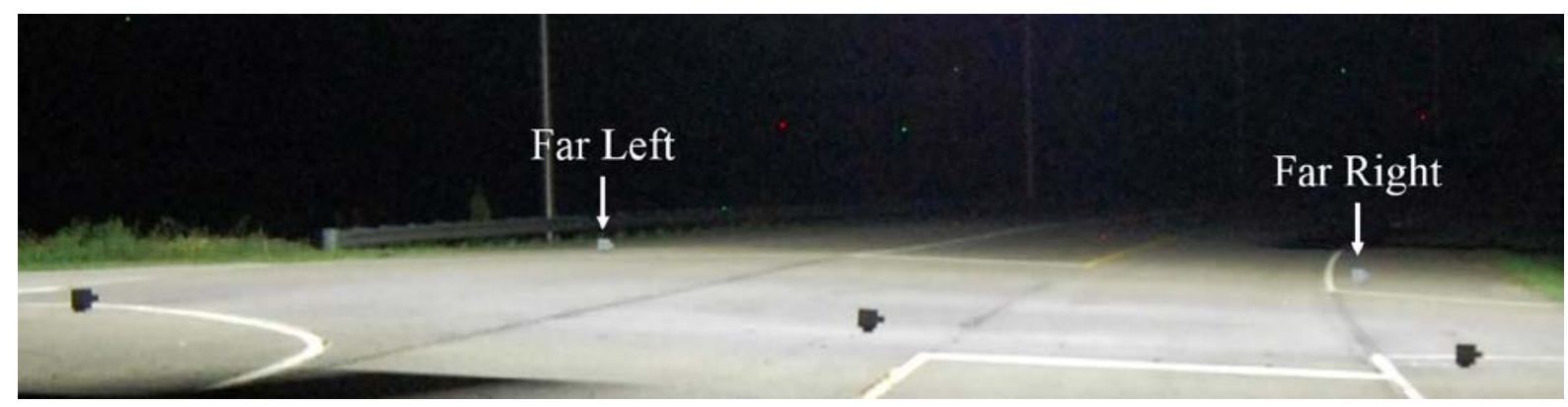

Figure 13. Photo of the intersection, illustrating background luminance values at the far left and far right target locations.

Increases in illuminance generally resulted in increased visual performance, consistent with earlier evidence on the effects of illuminance on visual performance (Boyce 1973, Eloholma, Ketomäki, Orreveteläinen and Halonen 2006, Terry and Gibbons 2015, Van Bommel and Tekelenburg 1986). Of these four noted studies, though, only the one by Terry and Gibbons (2015) used target detection distance as measure of visual performance and the one by Van Bommel and Tekelenburg (1986) used pedestrian detection distance as measure of visual performance. Terry and Gibbons (2015) evaluated ten incremental illuminances in a real driving scenario and showed that an increase in illuminance resulted in an increase in detection distance. However, their study did not explore the relationship between illuminance and potential plateaus in visual performance. Van Bommel and Tekelenburg (1986) studied the effect of three incremental luminance conditions on the detection distances of pedestrians and showed that increase in the luminance resulted in increased detection distance and this happened up to a luminance of $3.4 \mathrm{~cd} / \mathrm{m}^{2}$. The two remaining studies were conducted in laboratories and used reaction time as a measure of visual performance in a stimulus detection task.

Here, the increase in visual performance with increasing illuminance was not consistent across the three evaluated lighting configurations, being highest in the Box configuration. Further, even 
at the highest illuminance (21 lux), some target locations (near left in Both; near middle, near left, and far left in Approach) had mean detection distances that were shorter than the safe stopping distance in the Approach and Both lighting configurations. In the Box lighting configuration, though, all targets locations had mean detection distances longer than the safe stopping distance at an illuminance of $\geq 12$ lux. From this, it can be concluded that the Box lighting configuration illuminates the range of intersection target locations better than the other two lighting configurations, and at a lower illuminance.

Comparison of illuminances under each lighting configuration and target location indicated plateaus in visual performance in some conditions. However, only the Box lighting configuration showed a visual performance plateau for all target locations (excepting the far right target), and this plateau was consistently at the 8 or 12 lux illuminances (average intersection illuminance between 7 and 10 lux). The Approach and Both lighting configurations did not show consistent plateauing of visual performance for most of the target locations; for the few target locations where plateaus were evident, it varied between 16 and 21 lux. The lack of evidence of such plateaus in either the Both or Approach lighting configurations suggests that higher illuminances than tested are required to attain maximal visual performance.

The effect of illuminance on intersection visibility in this study did not completely align with earlier results on intersection visibility. Minoshima, Oka, Ikehara and Inukai (2006) reported that a mean roadway surface illuminance of 10 lux or higher will increase the visibility of the intersection irrespective of the lighting configuration, clearly in contrast with the present results. Only in the Box lighting configuration were participants able to detect all targets from a safe distance at an average intersection illuminance of 10 lux. In both the Approach and Both lighting 
configurations, and even at highest illuminance (12 lux in Approach and 13 in Both), none of the targets had mean results that were shorted that the safe distances. This discrepancy could be attributed to the different experimental methodologies used, in that visual performance here was objectively assessed (using detection distance) whereas Minoshima, Oka, Ikehara and Inukai (2006) used subjective ratings of intersection visibility.

The effect of illuminance on intersection visibility in this study could also translate lowering the night-to-day (ND) crash ratios at rural intersections. Bullough, Donnell and Rea (2013) calculated the RVP scores of different kinds of intersections and correlated them to ND crash ratios; they showed that rural intersections with a mean intersection illuminance of 10 lux reduces the ND crash ratio by about $2 \%$. Our results showing a plateauing of visual performance at the 10 lux mean horizontal illuminance, supports the results of Bullough, Donnell and Rea (2013). Edwards (2015) found that intersections with mean illuminance between 5 and 18.42 lux had fewer nighttime crashes than intersection with mean illuminance less than 5 lux. Although the illuminance range recommended by Edwards (2015) does not completely overlap the illuminance range (> 10 lux) where the visual performance plateaued here in the Box lighting configuration, there is, we believe, a strong indication that maintaining the mean illuminance of an intersection $\geq 10$ lux could result in lowering the number of nighttime crashes.

Age clearly influenced visual performance, with older group having shorter detection distances, and consistent with existing research on detection distances of targets and pedestrians that also found older drivers to have shorter detection distances (Bhagavathula and Gibbons 2013, Terry and Gibbons 2015) . A decrease in visual performance among the older participants is likely consequent to age-related physiological changes in the eyes that leads to reduced visual acuity 
and contrast sensitivity (Derefeldt, Lennerstrand and Lundh 1979, Evans and Ginsburg 1985, Joulan, Brémond and Hautière 2015, Owsley, Sekuler and Siemsen 1983, Salvi, Akhtar and Currie 2006). Interestingly, age-related differences in visual performance existed and were fairly similar in all three intersection lighting configurations. Both age groups had longer detection distances in the Box lighting configuration than either the Approach or Both configurations, indicating that the Box lighting configuration offers better visual performance for a wider range of drivers.

Headlamp beam patterns also affected the detection rates of targets. Targets on the left hand side of the road had lower detection rates especially in the no lighting condition (or headlamp only condition). We believe, this is because of the headlamp beam patterns which are tailored to reduce light output to the left lane to reduce glare to oncoming drivers. Targets on the right side of the road, in contrast, had a higher detection rate because of higher light output in that direction. On the left side, the far left target had a higher detection rate than the near left target, which we believe could be because of the lower background luminance of the far left target and that increased its contrast relative to that of the near left target, as mentioned above.

The results of this study have several practical implications. The observed differences in visual performance across the three lighting configurations imply that the part of the intersection that is illuminated plays a critical role in the visibility of targets at that intersection. For instance, illuminating the intersection box enhances the likelihood that targets at a variety of locations (e.g., intersection entry, exit, and the middle of the crosswalk) are visible from at least minimum safe stopping distance at $56 \mathrm{~km} / \mathrm{h}$ (35 mi/h). The Box lighting configuration has an additional benefit in that it requires only one luminaire to illuminate the entire intersection, whereas the 
other two configurations need at least as many luminaires as they are approaches at the intersection. A plateau in visual performance plateau was also evident for the Box lighting configuration, attained between 8 and 12 lux (mean intersection illuminance between 7 and 10 lux) depending on the target location. With the Box illuminated, increases in the illuminance beyond 12 lux (mean intersection illuminance 10 lux) are thus considered unlikely to substantially increase driver visual performance at an intersection. This mean horizontal illuminance of 10 lux in the Box lighting configuration is lower than existing specifications of 15 lux for "Major/Collector” or 13 lux for “Major/Local” functional classifications by IESNA's RP8, which closely resemble rural intersections in the United States. The Box lighting configuration also increased visual performance benefits to participants in both the younger and older age ranges, suggesting that a single configuration can be of benefit to a wide range of drivers. Use of Box lighting is thus argued as an effective approach to facilitate the development of intersection lighting design standards that will increase driver visual performance without over-lighting intersections. The need for a single luminaire and the noted performance plateaus further suggest that Box lighting can used to facilitate potential energy savings. However, this recommendation of illuminating the intersection box would be more suitable for installing lighting at intersections which currently do not have lighting. For intersections that are already illuminated, a cost-benefit analysis should be performed to compare the cost of installing a new lighting design (to illuminate the intersection box) versus the cost of installing new luminaires along with associated energy costs, to increase the illuminances to provide optimum visibility.

There are a few limitations of the current work that should be noted. First, there was no traffic (no additional vehicles) on the studied intersection and the signal lights at the intersection were turned off. These simplifications were used to reduce the possible confounding effects related to 
the presence of traffic and phase of the signal during approaches to the intersection. The presence of additional vehicles would also have introduced additional confounding effects of glare, which could also have affected the illuminance and target contrasts. The current experimental design was intended to isolate visual performance so that intersection lighting configurations and illuminances could be accurately evaluated. Second, pedestrians could not be used as objects for the detection task, as the length of the approach of the intersection used here was not long enough to show differences in the lighting configurations and illuminances. Third, the results of this study are mainly applicable to isolated or rural intersections with single source of illumination and which do not have continuous roadway lighting on any of the intersecting roads. Further, only one intersection approach was illuminated in the Approach and Both lighting configurations used here. In reality, all the approaches might be illuminated when Approach and Both configurations are used, which could substantially impact the luminance and contrast of objects located at the intersections and consequently their visibility. The presence of luminaires illuminating the approaches will increase object luminance at the intersections, however the change in object contrasts are difficult to predict as the objects will be illuminated from multiple directions. To address these limitations, future work should test visual performance under more realistic, complex scenarios with continuous lighting, and incorporate objective measures of pedestrian visibility to better determine the effectiveness of intersection lighting configurations.

\section{Conclusion}

In conclusion, driver nighttime visual performance at an intersection is clearly influenced the part of the intersection that is illuminated. The lighting configuration in which the intersection box was illuminated resulted in longer detection distances at every illuminance (other than no lighting). With the Box lighting configuration, visual performance also plateaued between a 
mean intersection illuminance of 7 and 10 lux, beyond this level additional increases in illuminance did not result in significant increases in visual performance (for both older and younger drivers). Lighting configurations in which only the intersection approach or both the intersection approach and box were illuminated performed worse than the Box lighting configuration, and did not show any consistent plateauing of visual performance. Younger participants had longer detection distances (by 29.3\%), and the influences of lighting configuration and illuminance were generally consistent between the two age groups studied. Results also showed that older drivers could benefit from higher illuminances (> 21 lux at Near Right target location or mean intersection illuminance of 12 lux), especially in the Approach and Both lighting configurations. These findings have important implications for lighting design of intersections, especially those at isolated/rural areas. Our results suggest that illuminating the intersection box can increase visual performance for the nighttime driver and could be an energy efficient solution.

\section{Funding}

Funding for this research was provided by the National Surface Transportation Safety Center for Excellence (NSTSCE). NSTSCE at VTTI develops and disseminates advanced transportation safety techniques and innovations. The mission of the center is to use state-of-the-art facilities, including the Virginia Smart Road, to conduct the research necessary to improve driver safety in both rural and urban communities. Stakeholders of NSTSCE include: Federal Highway Administration, Federal Motor Carrier Safety Administration, VDOT and the Virginia Center for Transportation Innovation and Research, General Motors Corporation, and Travelers Insurance. 


\section{References}

AASHTO. 2011. A policy on geometric design of highways and streets: 2011 Washington, D.C: American Association of State Highway and Transportation Officials.

Adrian W. 1989. Visibility of targets: Model for calculation. Lighting Research and Technology.21:181188.

Aulhorn E. 1964. Über die Beziehung zwischen Lichtsinn und Sehschärfe. Albrecht v Graefes Arch Ophthal. 1964/01/01;167:4-74.

Bhagavathula R, Gibbons RB. Role of Expectancy, Motion and Overhead Lighting on Nighttime Visibility. Proceedings of the Proceedings of the Human Factors and Ergonomics Society Annual Meeting; 2013: SAGE Publications.

Bhagavathula R, Gibbons RB, Edwards CJ. Effect of Static and Moving Objects on Driver Eye Movements and Detection Distances. Proceedings of the Transportation Research Board 91st Annual Meeting; 2012.

Bhagavathula R, Gibbons RB, Edwards CJ. 2015. Relationship between roadway illuminance level and nighttime rural intersection safety. Transportation Research Record: Journal of the Transportation Research Board.8-15.

Boyce P. 1973. Age, illuminance, visual performance and preference. Lighting Research and Technology.5:125-144.

Bullough J, Donnell E, Rea M. 2013. To illuminate or not to illuminate: Roadway lighting as it affects traffic safety at intersections. Accident Analysis \& Prevention. 4/1/;53:65-77.

CIE. 2010. CIE 115:2010 Lighting of Roads for Motor and Pedestrian Traffic. In: Commission Internationale de L'Eclairage.

Derefeldt G, Lennerstrand G, Lundh B. 1979. Age variations in normal human contrast sensitivity. Acta Ophthalmologica.57:679-690.

Donnell ET, Porter RJ, Shankar VN. 2010. A framework for estimating the safety effects of roadway lighting at intersections. Safety Science.48:1436-1444.

Edwards C. 2015. Lighting Levels for Isolated Intersections Leading to Safety Improvements. UoM Center for Transportation Studies.

Edwards CJ, Gibbons RB. 2008. Relationship of Vertical Illuminance to Pedestrian Visibility in Crosswalks. Transportation Research Record: Journal of the Transportation Research Board.2056:9-16.

Eloholma M, Ketomäki J, Orreveteläinen P, Halonen L. 2006. Visual performance in night-time driving conditions. Ophthalmic and Physiological Optics.26:254-263.

Evans DW, Ginsburg AP. 1985. Contrast sensitivity predicts age-related differences in highway-sign discriminability. Human Factors: The Journal of the Human Factors and Ergonomics Society.27:637-642. 
Fotios S, Qasem H, Cheal C, Uttley J. 2016. A pilot study of road lighting, cycle lighting and obstacle detection. Lighting Research and Technology.1477153515625103.

Gibbons R, Flintsch A, Williams B, Du J, Rakha H. 2013. Evaluation of Impact of Modern Headlamp Technology on Design Criteria for Sag Vertical Curves. Transportation Research Record: Journal of the Transportation Research Board.65-73.

Gibbons RB, Edwards CJ, Bhagavathula R, Carlson P, Owens DA. 2012. Development of visual model for exploring relationship between nighttime driving behavior and roadway visibility features. Transportation Research Record: Journal of the Transportation Research Board.2298:96-103.

Hills BL. 1975. Visibility under night driving conditions: Part 2. Field measurements using disc obstacles and a pedestrian dummy. Lighting Research and Technology. December 1, 1975;7:251-258.

IESNA. 2005. RP-8-00 American National Standard Practice for Roadway Lighting. IESoN America. (RP-8-00).

Isebrands HN, Hallmark SL, Hans Z, McDonald T, Preston H, Storm R. 2006. Safety Impacts of Street Lighting at Isolated Rural Intersections - Part II. (MN/RC-2006-35).

Janoff M. 1992. The effect of headlights on small target visibility. Journal of the Illuminating Engineering Society.21:46-53.

Janoff MS. 1993. The Relationship between Small Target Visibility and a Dynamic Measure of Driver Visual Performance. Journal of the Illuminating Engineering Society. 1993/01/01;22:104-112.

Joulan K, Brémond R, Hautière N. 2015. Towards an Analytical Age-Dependent Model of Contrast Sensitivity Functions for an Ageing Society. The Scientific World Journal.2015.

Mayeur A, Bremond R, Bastien JMC. 2010. The effect of the driving activity on target detection as a function of the visibility level: Implications for road lighting. Transportation Research Part F: Traffic Psychology and Behaviour.13:115-128.

Minoshima O, Oka K, Ikehara K, Inukai N. Research on Accident Reduction by Intersection Lighting. Proceedings of the Road Engineering Association of Asia and Australasia (REAAA) Conference; 2006.

Murdoch JB. 2003. Illuminating engineering: from Edison's lamp to the LED: Visions Communications.

NHTSA. 2014. Traffic Safety Facts 2013. Washington, DC.

Owsley C, Sekuler R, Siemsen D. 1983. Contrast sensitivity throughout adulthood. Vision research.23:689-699.

Oya H, Ando K, Kanoshima H. 2002. A Research on Interrelation between Illuminance at Intersections and Reduction in Traffic Accidents. Journal of Light \& Visual Environment.26:1_29-21_34.

Pretto P, Chatziastros A. Changes in optic flow and scene contrast affect the driving speed. Proceedings of the Driving Simulation Conference Europe; 2006.

Rea, Ouellette M. 1991. Relative visual performance: A basis for application. Lighting Research and Technology.23:135-144. 
Rea MS. 2000. The IESNA lighting handbook: reference \& application: IESNA.

Rea MS, Bullough JD, Zhou Y. 2010. A method for assessing the visibility benefits of roadway lighting. Lighting Research and Technology. March 31, 2010.

Salvi SM, Akhtar S, Currie Z. 2006. Ageing changes in the eye. Postgraduate Medical Journal.82:581587.

Shinar D. 1985. The Effects of Expectancy, Clothing Reflectance, and Detection Criterion on Nighttime Pedestrian Visibility. Human Factors: The Journal of the Human Factors and Ergonomics Society. June 1, 1985;27:327-333.

Smadi O, Hawkins N, Aldemir-Bektas B. 2011. Roadway Lighting and Safety: Pase II - Monitoring Quality, Durability and Efficiency. I. (IHRB Project TR-601).

Terry TN, Gibbons RB. Road-Surface Contrast and Adaptation of Visibility of Low-Contrast Targets. Proceedings of the Transportation Research Board 94th Annual Meeting; 2015.

Turner S, Sandt L, Toole J, Benz R, Petten R. 2006. FHWA UNIVERSITY COURSE ON BICYCLE AND PEDESTRIAN TRANSPORTATION: STUDENT WORKBOOK. McLean, VA: FH Administration. (FHWA-HRT-05-133).

Van Bommel WJ, Tekelenburg J. 1986. Visibility research for road lighting based on a dynamic situation. Lighting Research \& Technology.18:37-39.

Wortman R, Lipinski M. 1974. RURAL AT-GRADE INTERSECTION ILLUMINATION. (UILU-ENG74-2011).

Zwahlen H, Schnell T. 1999. Visibility of Road Markings as a Function of Age, Retroreflectivity Under Low-Beam and High-Beam Illumination at Night. Transportation Research Record: Journal of the Transportation Research Board.1692:152-163. 